\title{
Memory for frequency as a function of environmental context
}

\author{
ED ECKERT, N. JACK KANAK, and RICK STEVENS \\ University of Oklahoma, Norman, Oklahoma
}

\begin{abstract}
Comparative or absolute judgments of word-presentation frequency were studied in two experiments involving proactive-interference (PI) and retroactive-interference (RI) designs, manipulation of same and different environmental contexts for list presentation, and manipulation of same and different environmental contexts (relative to the critical list) for the frequency-judgment task. Comparative judgments yielded both PI and RI. Absolute judgments produced not only PI and RI, but also an interaction with the two context factors. PI and RI groups judged most accurately when the two lists had been presented in different contexts and judgment took place in the critical list context, and when the two lists had been presented in the same context and judgment was in a neutral context. Poorest performance occurred when presentation context was the same and judgment occurred in the same context, and when presentation contexts were different but judgment was in the nontarget list context.
\end{abstract}

The present studies are concerned with retroactiveinterference (RI) and proactive-interference (PI) effects in the judgments of word event frequency and the potential interactions of these effects with same versus different environmental contexts for the two tasks. The study is of relevance to several current issues in cognitive psychology, including the view that event frequency is automatically processed (Greene, 1984; Hasher \& Chromiak, 1977; Hasher \& Zacks, 1979; Hockley, 1984, Experiment 3), whether interference effects occur in recognition memory (Bowles \& Glanzer, 1983; Willis \& Underwood, 1983), and the influence that associations formed between list words and environmental stimuli have upon memory processes.

The influence of environmental context on memory, despite its long existence as a process postulated to be important to memory phenomena (e.g., McGeoch, 1942), has become the object of systematic research only relatively recently, despite earlier occasional forays (e.g., Abernethy, 1940; Bilodeau \& Schlosberg, 1951; Greenspoon \& Ranyard, 1957) and at least one attempt at theoretical integration (McGovern, 1964). The finding that information presented in one environment is best retained in memory if testing occurs in the same, rather than a different, environment has come to be termed the environmental-context effect. This effect has been observed in free-recall experiments (e.g., Godden \& Baddeley, 1975; Smith, Glenberg, \& Bjork, 1978; Smith, $1979,1982)$, in differential effects on free recall for different serial positions (Dolinsky \& Zabrucky, 1983; Nixon \& Kanak, 1981), in judgments of temporal duration (Block, 1982), in memory for spoken information (Rothkopf, Fisher, \& Bittington, 1982), in studies of proactive

Reprint requests should be addressed to N. Jack Kanak, Department of Psychology, University of Oklahoma, Norman, OK 73019. inhibition in classical serial learning (Dallett \& Wilcox, 1968), and in animal conditioning (Jobe, Mellgren, Feinberg, Littlejohn, \& Rigby, 1977; Wickens, Tuber, \& Wickens, 1983). Attempts to demonstrate the effect in recognition memory experiments have been generally unsuccessful (e.g., Godden \& Baddeley, 1980; Smith et al., 1978).

The current experiments were designed to test the accuracy of judgments of word event frequency within PI and RI designs as a function of same versus different environmental contexts for presentation of the target and nontarget lists and as a function of the same or different judgment test room contexts (target or nontarget). It was expected that, under PI and RI conditions, competing contextual associations developed between the context and target and nontarget items, presented within the same environmental context, would create the conditions for context-item associative interference with the accuracy of frequency judgments. Such context-based interference would not be expected to occur under the different-context presentation conditions.

In analogy with the views of McGovern (1964) regarding context/response-item competition between two different paired-associate lists, incidental associations formed between contextual stimuli and list items (and their frequency tags) of two different lists presented in the same context are predicted to enter into a classical interference relationship involving common stimuli (same context) and different responses (neutral words in two lists and differing frequency of occurrence of those words). This should produce a decrease in the accuracy of frequency-ofoccurrence judgments, relative to the different presentation context conditions, with this effect being strongest when both the two list presentation contexts and the judgment task contexts are the same.

Our view of the processes governing the influence of 
contextual associations assumes (1) that they are a relatively pure form of incidental learning without awareness, (2) that contextual cues have "elicitation" properties comparable to the conditioned stimulus (CS) in classical conditioning, (3) that this elicitation property influences the neural trace activation and availability of items (or, in this case, of item frequency tags), and (4) that contextual associations are governed by the laws of associative learning, including strengthening with practice/repetition and inhibition, facilitation, extinction, spontaneous recovery, and generalization/differentiation. The development of contextual associations and their functions, without awareness, may be viewed as a form of "automatic processing" and "automatic retrieval," with the retrieval system being subject to the laws of associative facilitation and inhibition. Our view that contextual associations influence cognitive processes without awareness and possess elicitation properties is not without implicit foundation in the literature. Marcel (1983) provided evidence to suggest that concept activation and spread of activation to semantically related concepts proceeds to affect memory independently of a subject's intentions or conscious awareness.

Experiment 1 reports the results of a comparative judgment task involving pairs of words of differing frequencies of presentation. Experiment 2 involves the use of an absolute-frequency judgment task of items presented singly. The basic features of the methodology of the two experiments were otherwise identical.

\section{METHOD}

\section{Design and Subjects}

The design for each experiment was a 2 (PI and RI) $\times 2$ (experimental and control) $\times 2$ (same or different list presentation context) $\times 2$ (judgment presentation context same as or different from critical list presentation context) factorial. The critical list was, of course, the second list presented to subjects in the PI condition, the first list for subjects in the RI condition, and the only list presented to the control subjects. The context manipulation of the judgment task resulted in the task's being performed either in the same context in which the critical list had been presented (a same-same or different-same combination of presentation and judgment contexts) or in a context different from that in which the critical list had been presented (same-different and different-different combinations of contexts). With these combinations labeled as SS, SD, DS, and DD for the two contextual (presentation and judgment, respectively) manipulations, the best performances should be produced by the DS and SD (neutral judgment context) and the poorest by the SS and DD (context of nontarget list) conditions. In the SD condition, involving a neutral room for judgment, neither context-item facilitation nor interference should occur at the time of judgment, such that judgment accuracy should be affected only by competition-produced unlearning of context-item frequency information during second-list presentation.

In addition to the 16 treatment conditions, a number of control precautions were exercised through counterbalancing. Half of the subjects in each treatment condition were administered one of two critical lists differing in item content, which in turn were completely crossed with respect to the two different lists, but random with respect to the rooms. Furthermore, two serial orders were completely crossed with respect to the two different lists, but random with respect to the rooms. The starting room was counterbalanced across subjects.

In each experiment, there were 192 introductory psychology students who participated as an optional course requirment. All subjects were naive to the experiment and were assigned randomly to conditions in order of their appearance at the laboratory.

\section{Contexts}

The context in this experiment was manipulated by the use of two different presentation and/or judgment settings (i.e., two rooms). The first room measured $18 \times 12 \times 10 \mathrm{ft}$. This room contained two bookcases, two desks, two wastepaper baskets, a hard wooden chair for the subject, and a table upon which a memory drum was situated. The table and chair were placed in the center of the room away from the walls. The bookcases contained large quantities of printed matter, and papers were strewn about on the desk and the floor. The room contained other irrelevant visual stimuli, posters, maps, and calendars. This room was well illuminated by fluorescent ceiling lamps, and the temperature was held constant at $76^{\circ} \mathrm{F}$.

The second room also measured $18 \times 12 \times 10 \mathrm{ft}$, but it was partitioned into an $8 \times 5 \times 10 \mathrm{ft}$ cubicle by means of three large bookcases. This cubicle contained only a padded chair and a small table. A small table lamp and a memory drum were the only articles present on the table. The room walls that were visible were bare, and the backs of bookcases formed the remaining walls of the cubicle. The partitioned area was illuminated only by a single $25-\mathrm{W}$ incandescent bulb desk lamp focused on the memory drum. The temperature was held constant at $68^{\circ} \mathrm{F}$.

\section{Lists}

Thirty-six words of AA frequency were chosen from the Thorndike and Lorge (1944) norms for use in the experiments. All of the words were nouns that ranged in length from four to seven letters. The words were relatively homogeneous with respect to imagery (Paivio, Yuille, \& Madigan, 1968) and were not associatively related (Palermo \& Jenkins, 1964). Half the words were randomly assigned to List A, and half to List B. Six of the words in each list were then selected randomly to appear six times, six to occur four times, and six to occur two times, making a total of 72 discrete presentations in each list. The order of the words within a list was random, with the restriction that repetitions of a given word be separated by at least two different words. In addition to the two different lists, two serial orders of each list were constructed.

\section{Procedure}

Before the lists were presented, the subjects were instructed to view and pronounce each word, presented for $2 \mathrm{sec}$ via a memory drum. They were told that some words would be repeated and that they should try to remember all of the words. No information about a retention task was given. After presentation of the first list, the subjects in the inhibition experimental groups viewed a second list $30 \mathrm{sec}$ later in either the same room or the other (different) room. The interlist interval was held constant at $30 \mathrm{sec}$ for the subjects in the same-context condition so that the interlist interval for the subjects who had to change rooms was equal. To control for disruption effects, the same-context-condition subjects were asked to step outside the room between tasks. The instructions for the second list were essentially the same as those for the first and required approximately $15 \mathrm{sec}$ to deliver. The time required to change rooms was $15 \mathrm{sec}$. After the second list had been presented, the inhibition experimental groups received the comparative (Experiment 1) or absolute (Experiment 2) judgment task either in the same context in which the critical list had been presented or in a different context. The intertask interval was held constant at $40 \mathrm{sec}$.

The subjects in the inhibition control groups received essentially the same treatment, except that a number-cancellation task either preceded or succeeded the presentation of the critical list. The control subjects performed the number-cancellation activity for an interval equal to the interval required by experimental group subjects to view a single word list $(114 \mathrm{sec})$. The intertask intervals for the control groups were equal to those of the experimental groups. The room of list presentation for the control subjects counterbalanced the room of critical list presentation for experimental subjects.

The comparative judgments of frequency were made on a sheet of paper containing 12 word pairings that represented each word from the appropriate list only once and six words that did not occur in any of the lists. Words having equal situational frequencies were not paired; however, every frequency (zero, two, four, and six) was paired with every other frequency twice. In Experiment 2, involving absolutefrequency judgments, the subjects were asked to make absolute judgments 
of the frequency of presentation of 24 words (six buffers), which were exposed one at a time.

\section{RESULTS AND DISCUSSION}

\section{Experiment 1}

A 2 (RI vs. PI Groups) $\times 2$ (experimental vs. control groups) $\times 2$ (presentation contexts) $\times 2$ (judgment contexts) between-groups analysis of variance was performed on the errors made in the comparative judgments of frequency. Significantly more errors were made by the experimental groups than by the control groups $[\mathrm{F}(1,176)=24.54, \mathrm{p}<.001, \mathrm{MSe}=1.19]$. The means (and standard deviations) for the experimental and control groups were $1.96(1.21)$ and $1.18(.940)$, respectively. No other significant main effects or interactions were obtained. Both PI and RI of comparative judgments were obtained to an equal degree, but neither context manipulation affected this recognition task.

\section{Experiment 2}

The absolute deviations from actual frequency of occurrence were calculated for each word and then summed to provide a score for each subject. The experimental groups (both PI and RI) were significantly less accurate than their respective control groups $[F(1,176)=26.72$, $\mathrm{p}<.0001, \mathrm{MSe}=47.19$ ], with overall deviation means (and standard deviations) of 30.45 (7.57) and 25.32 (5.53), respectively. There was no main effect of the PI$\mathrm{RI}$ factor and no interaction with any other variable, which leads to the conclusion that PI and RI effects were equal in magnitude. The occurrence of RI and PI in both experiments may be viewed as inconsistent with theoretical views holding that memory for frequency of occurrence of events is automatically processed (Hasher \& Chromiak, 1977; Hasher \& Zacks, 1979).

The expected three-way interaction of experimentalcontrol groups, presentation context, and judgment context was significant $[\mathrm{F}(1,176)=5.55, \mathrm{p}<.02]$. The means and standard deviations are present in Table 1. None of the single-list control conditions interacted significantly with either of the context variables, and individual comparisons revealed no significant differences among the control means. Although differences favoring "same" context are predicted by the theory, the literature illustrating failures at demonstrating context effects

Table 1

Mean Cumulative Absolute-Frequency Judgments and Deviations from Actual Presentation Frequency

\begin{tabular}{ccc}
\hline & \multicolumn{2}{c}{ Presentation Context } \\
\cline { 2 - 3 } E-C Condition & Same & Different \\
\hline \multirow{3}{*}{ E } & "Same" Judgment Context \\
C & 33.83 & \\
& 23.91 & 28.29 \\
E & "Different" Judgment Context & \\
C & 28.00 & 31.67 \\
& 25.41 & 26.67 \\
\hline
\end{tabular}

in simple recognition tasks is consistent with the present finding. Presumably a boundary condition of task difficulty, satisfied by the contextual association interference in the experimental groups, exists for context effects to be obtained in a frequency-judgment task.

The interaction of the two context factors with the experimental groups provides support for some of the predicted effects. The least accuracy was demonstrated by the two conditions expected to produce the poorest performance-the SS (mean=33.83) and DD (mean=31.67) conditions. In the SS condition, frequency information for two different lists becomes associated with the same context, which results in competition between interlist contextual associations, which is maximal when the PI or RI critical list judgment task also occurs in the same context. In the DD condition, independent context-item/ frequency-tag associations are developed for the two lists as a result of differing presentation contexts, but the judgment test occurs in the competing, noncritical list context in which frequency tags have also become associated with other words. These conditions did not differ from each other, but both were significantly inferior $(p<.05)$ to the DS and SD conditions.

The DS condition $($ mean $=28.29)$ was predicted to produce good performance, since the presentation of the two lists in different contexts allows the establishment of independent contextual associations and, furthermore, the judgment task occurs in the context in which the critical list was presented, allowing facilitation from contextitem/frequency-tag associations. The SD condition $($ mean $=28.00)$ involves the development of competing context-item/frequency-tag associations for the two lists, but the judgment task occurs in a neutral room so that neither contextual interference nor facilitation is operative at the time of the judgment. The fact that this neutral judgment context condition resulted in performance equaling that in the DS condition is somewhat surprising. One might expect RI but not PI (as a result of recency for the SD condition), yet both effects were found to be significant relative to their single-list controls. We offer one hypothesis that could explain the strong performance in the SD condition. In the absence of contextitem/frequency-tag competition at the time of the retention task, the subjects in the neutral judgment condition simply may have been able to access frequency tags independently for each list by memory for "response sets" of items/frequency tags for each list, since only List 1 or List 2 items were presented in the judgment task. Such an independent retrieval process is prevented in the SS condition by virtue of the activation of competing contextual associations from two lists with common frequency tags. For the DS condition, context-item/frequency-tag associations are already independently encoded at the time of presentation, and the judgment task is performed in the facilitating (critical list) context. Independent encodings also occur for the DD condition, but competition is activated at the time of the retention task by virtue of the judgment task's occurring in the presentation context of the noncritical list. 


\section{GENERAL DISCUSSION}

These experiments have demonstrated that both comparative- and absolute-frequency judgments are sensitive to PI and RI effects and thus represent findings germane to a current controversy regarding interference effects in recognition memory (cf. Bowles \& Glanzer, 1983; Willis \& Underwood, 1983). It is worth noting that in both experiments all significant effects were replicated in a measure of confidence ratings on the frequency tasks. In addition, the finding of context effects for absolute-frequency judgments, but not for comparative judgments, suggests that an element of retrieval may be necessary if a recognition task is to be sensitive to environmental-context manipulations. The absolute judgment task meets that criterion by requiring the "recall" of a frequency tag rather than a mere decision regarding which of two items appeared more frequently. The findings of RI and PI in both judgment tasks also argue against the position of Hasher and colleagues (Hasher \& Chromiak, 1977; Hasher \& Zacks, 1979) that memory for event frequency is automatically processed. The finding that absolute-frequency judgments are also sensitive to manipulations of the environmental context presents an additional problem for the "automatic processing" point of view. Rather, it could be argued that contextual associations undergo automatic processing and automatic elicitation in the presence of the contextual cues.

The obtained interaction of environmental-context manipulations with RI and PI conditions on the absolute judgment task represents a significant empirical and theoretical extension of recent research on the environmental-context phenomena. Interference with frequency judgments can be diminished when differential contexts are associated with two highly similar tasks. The data suggest that environmental-context associations can be critical to both retention and forgetting in tasks beyond those that are traditional "learning" tasks. It seems apparent that a systematic incorporation of environmental-context phenomena into a revised interference theory of forgetting may bring substantially greater precision to that theory. Furthermore, the practical relevance of the study of environmental-context effects assumes major importance as the phenomenon associated with such manipulations is shown to embrace a rapidly increasing array of processes and tasks.

\section{REFERENCES}

ABERNEThy, E. M. (1940). The effect of changed environmental conditions upon the results of college examinations. Journal of Psychology, 10, 293-301.

BilodeAu, I. M., \& SCHLOSBERg, H. (1951). Similarity in stimulating conditions as a variable in retroactive inhibition. Journal of Experimental Psychology, 41, 199-204.

BLOCK, R. A. (1982). Temporal judgments and contextual change. Journal of Experimental Psychology: Learning, Memory, and Cognition, 6, 530-544.

Bowles, N. L., \& Glanzer, M. (1983). An analysis of interference in recognition memory. Memory \& Cognition, 11, 307-315.

Dallet, K., \& Wilcox, S. G. (1968). Contextual stimuli and proactive inhibition. Journal of Experimental Psychology, 78, 475-480.

DOLINKSY, R., \& ZABRUCKY, K. (1983). Effects of environmental context changes on memory. Bulletin of the Psychonomic Society, 21, 423-426. Godden, D. R., \& Baddeley, A. D. (1975). Context-dependent memory in two natural environments: On land and underwater. British Journal of Psychology, 66, 325-331.

Godden, D. R., \& BADDELEY, A. D. (1980). When does context influence recognition memory? British Journal of Psychology, 71, 99-104.

GREENE, R. L. (1984). Incidental learning of event frequency. Memory \& Cognition, 12, 90-95.

GreENSPOON, J., \& RANYARD, R. (1957). Stimulus conditions and retroactive inhibition. Journal of Experimental Psychology, 53, 55-59.

Hasher, L., \& Chromiak, W. (1977). The processing of frequency information: An automatic mechanism? Journal of Verbal Learning and Verbal Behavior, 16, 173-184.

HASHER, L., \& ZACKS, R. T. (1979). Automatic and effortful processes in memory. Journal of Experimental Psychology: General, 108, 356-388.

HocKLEY, W. E. (1984). Retrieval of item frequency information in a continuous memory task. Memory \& Cognition, 12, 229-242.

Jobe, J. B., Mellgren, R. L., Feinberg, R. A., Littlejohn, R. L., \& RIGBY, R. L. (1977). Patterning, partial reinforcements, and Nlength effects at spaced trials as a function of reinstatement of retrieval cues. Learning and Motivation, 8, 77-97.

MARCEL, A. J. (1983). Conscious and unconscious perception: An approach to the relations between phenomenal experience and perceptual processes. Cognitive Psychology, 15, 238-300.

MCGEOCH, J. A. (1942). The psychology of human learning. New York: Longman, Green.

McGoverN, J. B. (1964). Extinction of associations in four transfer paradigms. Psychological Monographs, 78, (16, Whole No. 593).

NIXON, S. J., \& KANAK, N. J. (1981). The interactive effects of instructional set and environmental context changes on the serial position effect. Bulletin of the Psychonomic Society, 18, 237-240.

Palermo, D. S., \& Jenkins, J. J. (1964). Word association norms. Minneapolis: University of Minnesota Press.

Paivio, A., Yuille, J. C., \& Madigan, S. (1968). Concretenes, imagery, and meaningfulness for 925 nouns. Journal of Experimental Psychology Monographs, 76(1, Pt. 2).

RothKopf, E. Z., Fisher, D. B., \& BitTington, M. J. (1982). Effects of spatial context during acquisition on the recall of attributive information. Journal of Experimental Psychology: Learning, Memory, and Cognition, 8, 126-138.

SMITH, S. M. (1979). Remembering in and out of context. Journal of Experimental Psychology: Human Learning and Memory, 5, 460-471.

SMITH, S. M. (1982). Enhancement of recall using multiple environmental contexts during learning. Memory \& Cognition, 19, 405-412.

SMith, S. M., GlenberG, A., \& BJoRK, R. A. (1978). Environmental context and human memory. Memory \& Cognition, 6, 343-353.

THORNDIKE, E. L., \& LORGE, I. (1944). The teacher's word book of 30,000 words. New York: Teacher's College, Columbia University Press.

Wickens, C., Tuber, D. S., \& Wickens, D. D. (1983). Journal of Experimental Psychology: General, 112, 41-57.

WILLIS, G. B., \& UNDERWOOD, B. J. (1983). A lack of interference effects in recognition memory. Bulletin of the Psychonomic Society, 21, 427-430.

(Revision received for publication September 24, 1984.) 